\title{
Imprint cytology: a useful screening test for diagnosis of Helicobacter pylori in resource poor settings
}

\author{
Piyumali Sandareka Arachchi ${ }^{1} \mathbb{0}$, Manjula Manoji Weerasekera $^{1}$, Bimalka Seneviratne ${ }^{2}$, Deepaka Weerasekera ${ }^{3}$,
} Neluka Fernando ${ }^{1}$ and Chinthika Prabhashinie Gunasekara ${ }^{1 *}$ (B)

\begin{abstract}
Objective: The study aimed to compare the usefulness of two staining methods for imprint cytology for diagnosis of Helicobacter pylori infection. Gastric biopsy specimens (from dyspeptic patients attending routine upper gastrointestinal endoscopy) were placed on glass slides to obtain imprints. The imprints were stained with Toluidine blue and Giemsa stains separately and observed under $\times 400$ magnification using a light microscope. Imprinted biopsies were sectioned and stained with $\mathrm{H} \&$ E stain and Giemsa stain for histological analysis. Diagnosis of H. pylori infection in both imprint and histological sections were confirmed by a consultant pathologist. The sensitivity, specificity, positive predictive value and negative predictive value of each stain were calculated and benchmarked against histological diagnosis.

Results: Of the 55 dyspeptic patients enrolled in the study, 5 were positive for $\mathrm{H}$. pylori by Toluidine blue stain and 4 by Giemsa stain. The sensitivity of Toluidine blue stain (57.1\%) was higher than Giemsa stain (42.9\%) while the specificity of both stains was equal (97.9\%). Giemsa stain gave a better discrimination for identification of H. pylori bacteria among the mucosal background. Imprint cytology is a rapid, simple and cost effective diagnosis method that can supplement histological diagnosis.
\end{abstract}

Keywords: Helicobacter pylori, Diagnosis, Imprint cytology, Toluidine blue, Giemsa

\section{Introduction}

Helicobacter pylori diagnosis is a challenging task despite the availability of several diagnostic methods. In Sri Lanka, histological investigations are a main approach for $H$. pylori diagnosis. A drawback of this approach is the long turnaround time incurred due to the laborious preparations involved in specimen processing [1]. Therefore a rapid low cost and simple method to diagnose $H$. pylori infection will enable initiation of treatment immediately.

Imprint cytology enables the visualization of $H$. pylori using a simple staining method. It can further complement histological diagnosis of $H$. pylori. Studies

\footnotetext{
*Correspondence: chinthika@sjp.ac.lk

${ }^{1}$ Department of Microbiology, Faculty of Medical Sciences, University

of Sri Jayewardenepura, Gangodawila, Nugegoda, Sri Lanka

Full list of author information is available at the end of the article
}

investigating the diagnostic utility of imprint cytology for $H$. pylori report high sensitivity of $(83 \%)$ and specificity (100\%) [2]. Further combination of imprint cytology with histology can improve the accuracy of diagnosis to $100 \%$ [3-5]. However currently imprint cytology is rarely used in H. pylori diagnosis.

Isolation of $H$. pylori for microbiological diagnosis is difficult due to the fastidious nature of the organisms which require special microaerophilic culture conditions $[6,7]$. However imprint cytology will enable microbiologists to identify $H$. pylori based on characteristic morphological appearance in the biopsy smear using commonly available stains thus facilitating an early diagnostic opportunity. This method offers a rapid, costeffective and simple diagnostic technique which can supplement the routine histological and biopsy urease tests as well as prove to be a valuable method which can be 
practiced in routine use in the diagnosis of $H$. pylori in developing countries.

\section{Main text \\ Methodology \\ Study population and specimens}

Dyspeptic patients attending routine upper gastrointestinal endoscopy at a tertiary care hospital in Sri Lanka were enrolled in the study after obtaining written informed consent. Patients who were less than 18 years of age, patients who were mentally unstable, those who have been on antibiotics for the past month and patients with malignant diseases (e.g., gastric cancer) were excluded from the study population. Fifty five patients were enrolled in the study and the socio-demographic and medical data were collected using an interviewer-administered questionnaire. From each patient, two biopsy specimens were collected for imprints and histology.

\section{Specimen processing}

The two biopsy specimens were placed on clean glass slides separately and imprints were obtained from each biopsy. After obtaining an imprint, each biopsy was placed in $10 \%$ formal saline (Welcome chemicals, Sri Lanka). The imprint slides were air-dried. The biopsy specimens were transported to the Department of Pathology at Faculty of Medical Sciences, University of Sri Jayewardenepura for histopathological investigations.

\section{Histopathological investigations}

The biopsy specimens were dehydrated using an alcohol gradient, embedded in paraffin wax and sectioned into four micron sections which were placed on clean glass slides. The sections were stained with Hematoxylin (Avondale, England) and Eosin (BDH, England) stain and Giemsa stain (LOBA chemie, India) before being graded according to the updated Sydney system [8] by a consultant pathologist.

\section{Imprint cytology of biopsy specimens}

After air-drying, one imprint specimen was flooded with 0.5\% Toluidine blue stain (Himedia, India) and washed with water after $1 \mathrm{~min}$ [9]. The second specimen was placed in a trough containing Giemsa working solution (LOBA chemie, India) and allowed to stain for $15 \mathrm{~min}$ before washing with running water [10]. The stained slides were dried and mounted using DPX mounting medium (Merck, India). The slides were observed under $\times 400$ magnification using a light microscope.

\section{Results and discussion}

The $H$. pylori infection was investigated in gastric biopsies taken from 55 dyspeptic patients (Additional file 3:
Book 1). Of these patients 34 were male while 21 were female with a median age of 56 (age range from 21-82). Of the 55 dyspeptic patients, 7 were diagnosed as $H$. pylori infected by histology. Five patients $(5 / 55)$ were diagnosed as $H$. pylori-positive by Toluidine blue stain while $4(4 / 55)$ were diagnosed with Giemsa stain.

Both Toluidine blue and Giemsa staining of the imprints revealed the strongly stained unique morphology of $H$. pylori visible as curved or spiral shaped rods embedded in the gastric mucosa. On comparison, Toluidine blue stained smears revealed the characteristic morphology of $H$. pylori. The meta-chromatic properties of this dye enable the differentiation of $H$. pylori from the mucus and the epithelial cells, where the bacteria stains dark blue against a variably blue background [11]. Toluidine blue staining enabled observation of both high (Fig. 1a) and low density (Fig. 1b) of H. pylori in the imprint smears. When slides containing gastric biopsy imprints were stained with Giemsa stain, the epithelial cells on the slide were visualized in blue color while the H. pylori bacteria were stained in magenta color which enabled better discrimination of the bacteria compared to Toluidine blue stain (Fig. 2). Giemsa being a multiple stain contains three dyes: methylene blue, Azure B and Eosin which contributes to better discrimination of bacteria from the background. By the Giemsa stain, the nuclear material of both bacteria and cells are stained dark blue to violet and the mucus is stained pale blue [12]. However Toluidine blue was more useful to determine the bacterial density compared to the Giemsa stain.

Considering the histological diagnosis of H. pylori as the benchmark, the sensitivity specificity, positive predictive value (PPV) and negative predictive value (NPV) of Toluidine blue stain and Giemsa stain are described in Table 1. Sensitivity of Toluidine blue staining for diagnosis of $H$. pylori infection was $57.1 \%$ while the specificity was $97.9 \%$. The PPV and NPV of Toluidine blue staining was 80.0 and $94.0 \%$ respectively, when compared with histological diagnosis. Diagnosis of $H$. pylori by Giemsa stain had a sensitivity of $42.9 \%$ and a specificity of $97.9 \%$ compared with histological diagnosis. The PPV and NPV of Giemsa stain was 75.0 and $92.2 \%$ respectively. The sensitivity of Toluidine blue stain was higher than Giemsa stain while both stains had similar specificity.

Of the seven specimens diagnosed as $H$. pylori-positive by histology, four specimens were positive for $H$. pylori by Toluidine blue stain while three were positive by Giemsa stain. Further, of $48 \mathrm{H}$. pylori negative (by histology) biopsy specimens $H$. pylori was identified in one specimen by both staining methods.

Of the seven $H$. pylori-positive specimens, three were diagnosed with high $H$. pylori density and the remaining four with low H. pylori density by histology. All three 


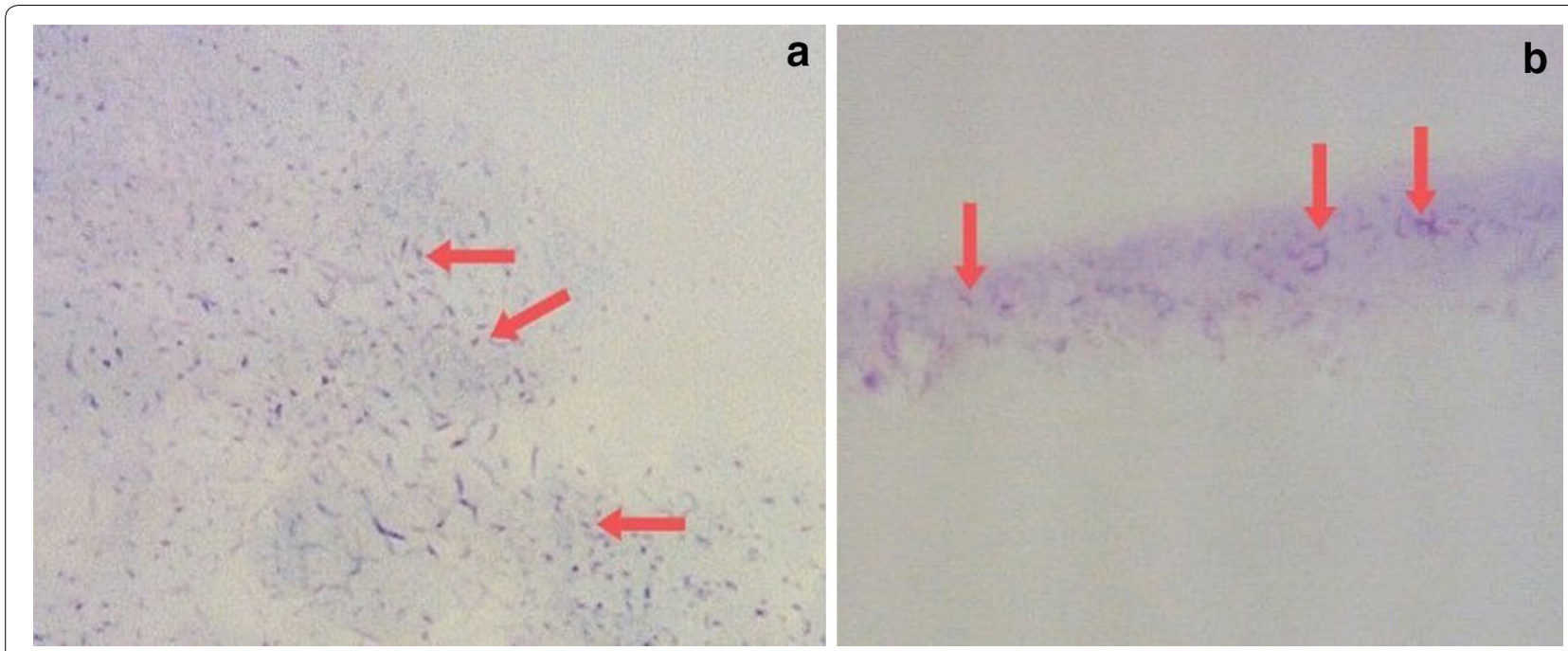

Fig. 1 Toluidine blue staining of H. pylori-positive specimens with their characteristic curved-spiral rod shape ( $\times 400$ magnification). a Imprint smear specimen with high density H. pylori; b Low density H. pylori in dark blue among mucus stained light blue

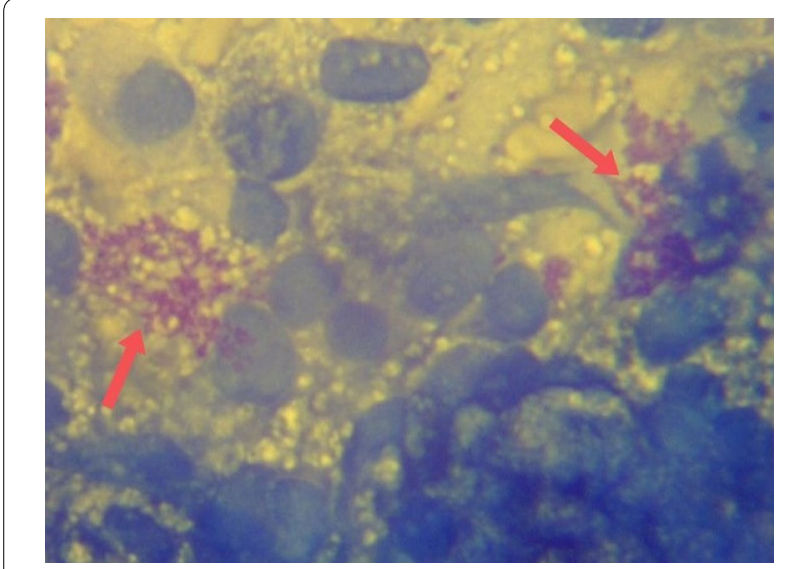

Fig. 2 Image of a H. pylori-positive imprint slide stained with Giemsa stain ( $\times 400$ magnification). Epithelial cells are stained in dark blue while H. pylori bacteria can be seen in magenta color

high density specimens were diagnosed as positive by both Toluidine blue and Giemsa stains. One low density specimen was diagnosed as positive by Toluidine blue stain but not by Giemsa stain. Low density specimens being diagnosed as $H$. pylori-negative by both stains may be due to poor transfer of bacteria from the biopsy specimen to the slide which may be a disadvantage of the imprint cytology method.

When considering a single biopsy specimen, imprint cytology was found to be a simple staining method which maximizes the use of this biopsy obtained for histological investigations without affecting the results of histology $[4,13]$. Thus the quality of the imprint as well as the integrity of the tissue which was sectioned needs to be high enabling histological review. Therefore during the preparation of imprints care should be taken to ensure that the biopsy specimen is undamaged by gentle handling by rolling rather than pressing hard on the biopsy specimen or dragging the specimen across the slide [13]. As the same biopsy specimen can then be used for histological investigations, use of imprint cytology can maximize the diagnostic utility of the biopsy specimen.

Several reports indicate a high sensitivity and specificity by imprint cytology [5, 14]. However, in this study while imprint cytology was $100 \%$ in agreement with high density $H$. pylori containing biopsy histology, the positivity for low density $H$. pylori containing biopsy was poor as has been reported by other study groups [4]. A disadvantage of imprint smear preparation is that the low

Table 1 Comparison of the two stains used for imprint cytology in the diagnosis of $\boldsymbol{H}$. pylori infection

\begin{tabular}{|c|c|c|c|c|c|}
\hline & Sensitivity (\%) & Specificity (\%) & $\begin{array}{l}\text { Positive predictive } \\
\text { value (\%) }\end{array}$ & $\begin{array}{l}\text { Negative predictive } \\
\text { value }(\%)\end{array}$ & $\begin{array}{l}\text { Kappa } \\
\text { coefficient }\end{array}$ \\
\hline Toluidine blue stain & 57.1 & 97.9 & 80.0 & 94.0 & 0.627 \\
\hline Giemsa stain & 42.9 & 97.9 & 75.0 & 92.2 & 0.499 \\
\hline
\end{tabular}


density specimens may be diagnosed as $H$. pylori negative due to poor transfer of bacteria from the biopsy specimen onto the slide. Further, processing or staining errors may reduce the sensitivity of the imprint cytology diagnosis by giving rise to artifacts in the imprint slide which may give false positive results as can be seen in this study where one specimen $H$. pylori-negative by histology was diagnosed as positive by both imprint stains.

While histology is widely used for diagnosis of $H$. pylori infection a major drawback of this approach is the need for sophisticated equipment and protocols as well as high technical skills of the laboratory personnel during the processing of the biopsy. In contrast imprint cytology can be carried out within $30 \mathrm{~min}$ of the collection of biopsy providing a tremendous advantage as therapy can be commenced on the same day before the patient leaves the endoscopy unit [2]. The histological investigations enable both the diagnosis of $H$. pylori infection as well as determination of the histological severity. However, imprint cytology cannot be used to provide information on mucosal inflammation, metaplasia, atrophy and other histological changes but will support the diagnosis of $H$ pylori.

Stool antigen test and Realtime PCR are several diagnostic methods that have also been introduced to diagnose the $H$. pylori infection. Both diagnostic methods have a high sensitivity and specificity in $H$. pylori diagnosis and can be carried out as non invasive assays. However, both these methods are expensive when compared with the imprint cytology method as these methods require specific equipment and expensive reagents to carry out the diagnosis.

Further this method is not labor intensive and facilitates rapid interpretation of results compared to $3-5$ days needed for the histological report. Both Toluidine blue and Giemsa stains can be prepared beforehand and applied directly to imprint slides. However, the Toluidine blue stain has to be stored at $4-8{ }^{\circ} \mathrm{C}$ when not in use while the Giemsa solution can be stored at room temperature. When considering the cost per test, both Toluidine blue stained and Giemsa stained imprint smears incurred a very low cost of approximately Rs. $5.00-10.00$ per slide. Therefore using imprint cytology as a screening test before performing histology will not increase the overall diagnostic cost significantly.

\section{Conclusion}

In conclusion, although imprint cytology alone is less sensitive than histology in diagnosis of H. pylori infection, it offers a rapid, economical and simple screening method which can supplement histological findings thus increasing the diagnostic sensitivity. Further the biopsy specimen used for imprint smear preparation can subsequently be used for the histological investigations thereby maximizing the diagnostic utility of the biopsy specimen. Increasing the number of specimens taken (from 2 to 5 ) and taking specimens from different places of the stomach for imprint cytology and histology may increase the accuracy of $H$. pylori diagnosis.

\section{Limitations}

The prevalence of $H$. pylori infection among the study population may have been affected by the use of PPI medication, as PPI use is reported to shift $H$. pylori from antrum to the corpus. The biopsy specimens were collected from the antrum of the patients' stomach which may have lowered the proportion of $H$. pylori infected patients.

As can be observed in the study, the bacterial density affects the sensitivity of the imprint cytology and specimens with low $H$. pylori density resulted were diagnosed as negative by imprint cytology.

During sample processing and staining, errors can cause the appearance of artifact which may lead to false positive diagnosis.

\section{Additional files}

Additional file 1: Figure S1. Image of a H. pylori-negative slide stained in Toluidine blue stain ( $\times 400$ magnification). Bacilli, stained in dark blue can be observed among the mucus and epithelial cells.

Additional file 2: Figure S2. H. pylori-negative imprint slide stained in Giemsa stain (x400 magnification).

Additional file 3: Book 1. Contains detailed results of all subjects enrolled for the study.

\section{Abbreviations}

NPV: negative predictive value; PPI: proton pump inhibitor; PPV: positive predictive value.

\section{Authors' contributions}

CPG, MMW and NF conceived the study. DW contributed with patient selection and sample collection. BS carried out the histopathological investigations. PSA designed the study protocol, carried out the laboratory work, and in addition carried out the statistical analysis and drafting of the manuscript. All authors read and approved the final manuscript.

\section{Author details}

${ }^{1}$ Department of Microbiology, Faculty of Medical Sciences, University of Sri Jayewardenepura, Gangodawila, Nugegoda, Sri Lanka. ${ }^{2}$ Department of Pathology, Faculty of Medical Sciences, University of Sri Jayewardenepura, Gangodawila, Nugegoda, Sri Lanka. ${ }^{3}$ Department of Surgery, Faculty of Medical Sciences, University of Sri Jayewardenepura, Gangodawila, Nugegoda, Sri Lanka.

\section{Acknowledgements}

The authors acknowledge the contribution made by the staff at the Endoscopy Unit of the Colombo South Teaching Hospital, staff at the Departments of Pathology and Microbiology, Faculty of Medical Sciences, University of Sri Jayewardenepura. 


\section{Competing interests}

The authors declare that they have no competing interests.

\section{Availability of data and materials}

The datasets generated and/or analysed during the current study are available from the corresponding author on reasonable request.

\section{Consent for publication}

Not applicable.

\section{Ethical approval and consent to participate}

Ethical approval for the study was obtained from the Ethics Review Committee, Faculty of Medical Sciences, University of Sri Jayewardenepura (Application no 14/15) and Colombo South Teaching Hospital, Kalubowila (Application no 450). Written informed consent was obtained from all participants before being enrolled in the study.

\section{Funding}

This work was supported by the National Science Foundation (NSF/ SCH/2015/04) and University of Sri Jayewardenepura Research Grants (ASP/01/RE/MED/2017/28).

\section{Publisher's Note}

Springer Nature remains neutral with regard to jurisdictional claims in published maps and institutional affiliations.

Received: 13 January 2018 Accepted: 12 July 2018

Published online: 16 July 2018

\section{References}

1. Patel S, Smith JB, Kurbatova E, Guarner J. Factors that impact turnaround time of surgical pathology specimens in an academic institution. Hum Pathol. 2012:43(9):1501-5.

2. Kaur G, Madhavan M, Basri AH, Hamid A, Sain M, Shaiful M, et al. Rapid diagnosis of Helicobacter pylori infection in gastric imprint smears. Southeast Asian J Trop Med Public Health. 2004;35(3):676-80.
3. Jafari M, Khalilian A, Rostampour F. Comparison the sensitivity of stomach mucus touching cytology and urease rapid test in Helicobacter pylori diagnosis in endoscopies patients with gastritis or peptic ulcer. Jundishapur J Microbiol. 2013;6(4):4-7.

4. Rahbar M, Mardanpur K, Tavafzadeh R. Imprint cutology: a simple cost effectiveness analysis for diagnosing Helicobacter pylori, in west of Iran. Med J Islam Repub Iran. 2012;26(1):12-6.

5. Misra SP, Dwivedi M, Misra V, Gupta SC. Imprint cytology — a cheap, rapid and effective method for diagnosing Helicobacter pylori. Postgr Med J. 1993;69:291-5.

6. Waidyarthne E, Mudduwa L, Lekamwasam J, Lekamwasam S. Helicobacter pylori detection techniques: comparison of sensitivity, specificity and cost. Gall Med J. 2012;17(2):1-7.

7. Kullavanijaya P, Thong-ngam D. Analysis of eight different methods for the detection of Helicobacter pylori infection in patients with dyspepsia. J Gastroenterol Hepatol. 2004;19:1392-6.

8. Price AB. The Sydney system: histological division. J Gastroenterol Hepatol. 1990;1991(6):209-22.

9. Chandra S, Chandra H, Sindhwani G. Role of rapid on-site evaluation with cyto-histopathological correlation in diagnosis of lung lesion. J Cytol. 2014;31(4):189-93.

10. Boldt MS, Pereira RD. Barbosa AJA. Histological identification of H. pylori stained by hematoxylin-eosin and giemsa: review for quality control. J Bras Patol Med Lab. 2015;51(2):108-12.

11. Sridharan G, Shankar AA. Toluidine blue: a review of its chemistry and clinical utility. J Oral Maxillofac Pathol. 2012;16(2):251-5.

12. Young B, Woodford $P, O^{\prime}$ Dowd $G$. Wheater's functional histology a text and colour atlas. 6th ed. London: Churchill Livingstone, Elsevier; 2014.

13. Misra SP, Misra V, Dwivedi M, Singh PA, Gupta SC. Diagnosing Helicobacter pylori by imprint cytology: can the same biopsy specimen be used for histology? Diagn Cytopathol. 1997;18(5):330-2.

14. Vijayanarasimha D, Mahadevappa A, Manjunath GV, Sunila R. Imprint cytology: a diagnostic aid in interpretation of upper gastrointestinal endoscopic biopsies. J Dig Endosc. 2014;5(17):144-8.
Ready to submit your research? Choose BMC and benefit from:

- fast, convenient online submission

- thorough peer review by experienced researchers in your field

- rapid publication on acceptance

- support for research data, including large and complex data types

- gold Open Access which fosters wider collaboration and increased citations

- maximum visibility for your research: over $100 \mathrm{M}$ website views per year

At BMC, research is always in progress.

Learn more biomedcentral.com/submissions 